\title{
AQUARIUS FINAL RELEASE PRODUCT AND FULL RANGE CALIBRATION OF L-BAND RADIOMETERS
}

\author{
Emmanuel P. Dinnat ${ }^{1,2}$, David M. Le Vine ${ }^{1}$, Liang Hong ${ }^{1,3}$ \\ ${ }^{1}$ NASA-Goddard Space Flight Center, Greenbelt, MD 20771, U.S.A. \\ ${ }^{2}$ Chapman University, Orange, CA 92866, U.S.A. \\ ${ }^{3}$ Science Applications International Corporation
}

\begin{abstract}
Aquarius final product V5.0 has been released. The dataset includes close to four years of global radiometric measurements at L-band. The mission's objective was to monitor sea surface salinity, but other applications of its data over land and the cryosphere have been developed. For this reason, it is important to have accurate calibration over the full range of antenna temperatures from natural targets. It is also needed in order to combine Aquarius measurements with other L-band sensors. Aquarius calibration is strongly focused on the ocean. We present a research product which is part of the final release and aims at producing an accurate calibration from the low end (celestial sky) to the high end (land and ice) of the brightness temperature scale. We calibrate the Aquarius radiometers using measurements over the Sky and oceans and assess the new calibration using measurements over land.
\end{abstract}

\section{Index Terms- Microwave, Radiometry, L-band, Aquarius, Calibration}

\section{INTRODUCTION}

Aquarius was a NASA spaceborne instrument that combined three radiometers and one scatterometer at Lband. It operated between Aug 25, 2011 and June 7, 2015 [1]. Because the goal of Aquarius was to measure sea surface salinity in the open ocean, the calibration of the instrument has been tuned to achieve this goal. The antenna temperature (Ta) is calibrated using the difference between global ocean Ta observation over a 7-day period and $\mathrm{Ta}$ simulations from a forward radiative transfer model [2] using a reference field for salinity. An independent check of the calibration and its temporal stability is performed using observations over the cold sky [3]. Because the forward model is consistent with the inverse model used in the salinity retrieval, this calibration procedure ensures that $\mathrm{Ta}$ is properly calibrated for the purpose of producing salinity maps (i.e. it is consistent with the reference field in term of global average). However, it can leave biases at the low (celestial sky) and high (land) ends of the Ta scale. For

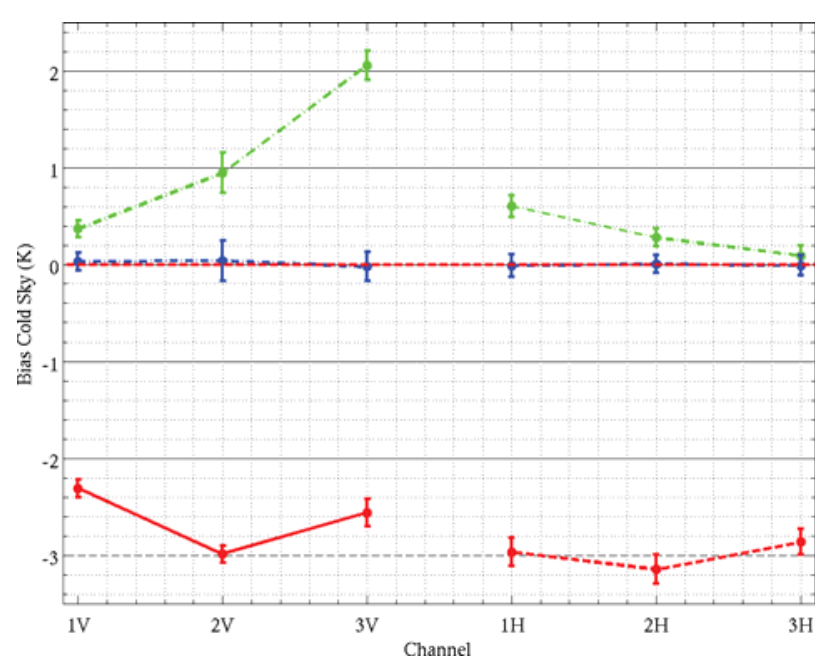

Fig. 1. Bias in Aquarius antenna temperature over the cold sky with channels reported in X-axis. Bias is computed as the difference between Ta measured by Aquarius and simulations over the celestial sky during the calibration maneuvers. Points represent the bias (median difference) and the error bars the standard deviation over 30 calibration maneuvers spread over more than 3 years. (Red) Aquarius V2.0 and simulation using numerical antenna pattern. (Green) V5.0 and Hybrid antenna pattern. (Blue) V5.1 and Hybrid antenna pattern.

example, before an error was corrected in the model for the antenna patterns [3], Aquarius had significant biases over the sky (Fig. 1, red curve) and land that did not impact the salinity product substantially. The use of cold sky calibration (CSC) and data at the warm end helped correct for the antenna pattern and mitigate the biases (Fig. 1, green curve). But some biases remain in the latest version (Fig. 1, green curve and Fig. 2), particularly for the channel $3 \mathrm{~V}$ which is $2 \mathrm{~K}$ too warm over the sky. This could be a problem for application of Aquarius data over land and the cryosphere [4]-[7], or for comparison and merging of Aquarius TB with other L-band instruments which use different calibration approach.

The final release of the Aquarius product includes a research product that aims at providing calibrated observation over the full range of natural Ta, namely between $\sim 5 \mathrm{~K}$ (cold sky) and $\sim 300 \mathrm{~K}$ (land). The standard product, version 5.0, uses essentially the ocean for its vicarious calibration. We 

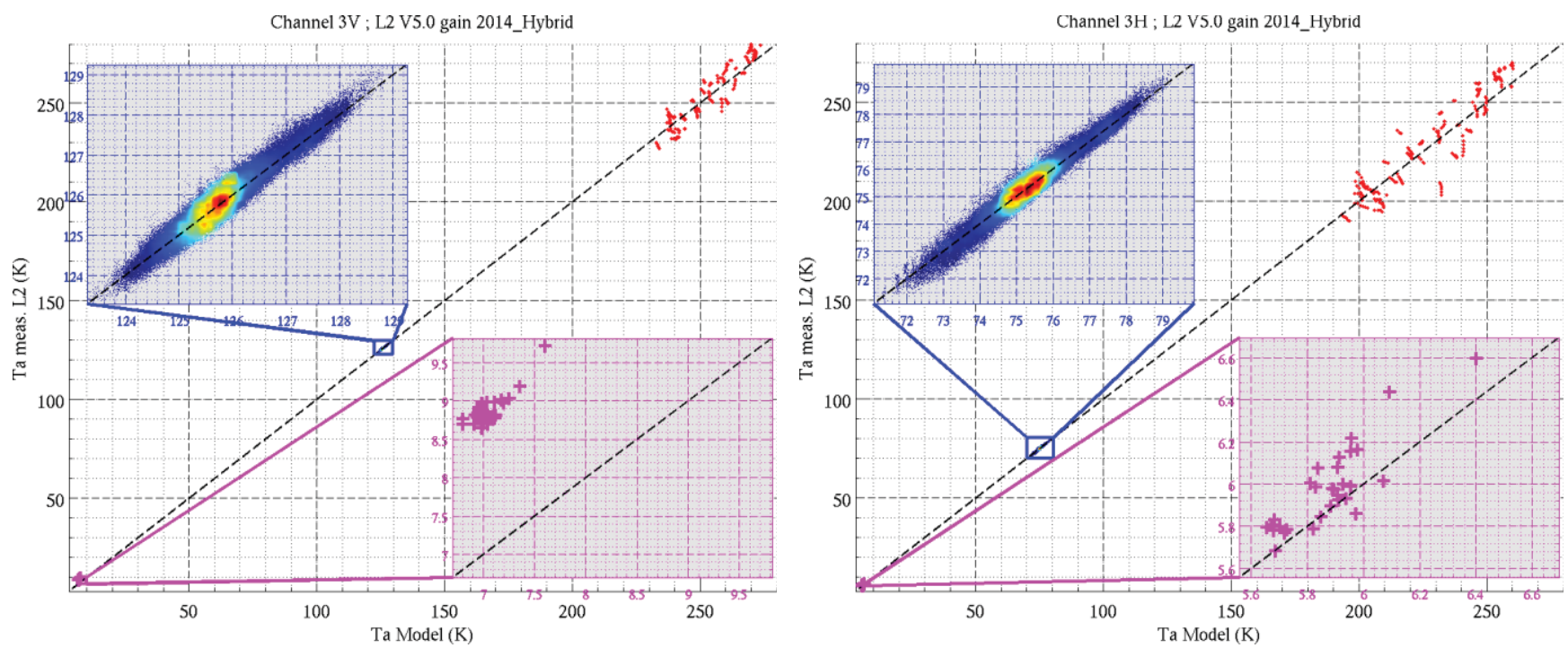

Fig. 2. $T_{a}$ measured by Aquarius versus simulated over (magenta inset) the Celestial Sky, (blue inset) ocean and (red dots) land, for the outer beam at (left) vertical and (right) horizontal polarizations. Insets are close-ups of ocean and Sky data. Aquarius observations from final release V5.0.

describe here the version 5.1 which includes an additional step to remove the bias at the cold end and keep Ta largely unchanged at the ocean.

\section{METHODS}

\subsection{Correcting the calibration}

To recalibrate antenna temperatures for V5.1, a linear regression is performed between Aquarius observations and a reference Ta obtained from simulations over the Sky and ocean. The recalibrated data are computed as

$$
T_{a, 5.1}=a \cdot T_{a, 5.0}+b,
$$

where $T_{a, 5.0}$ is the antenna temperature in the $\mathrm{L} 2$ product V5.0. Coefficients $a$ and $b$ are computed such that

$$
<T_{a, 5.1}>=<T_{a, s i m}>,
$$

where $T_{a, \text { sim }}$ are antenna temperatures simulated using a forward radiative transfer model [2]. The averages $<>$ are computed over the $30 \mathrm{CSC}$ for the low end and over thousands of observation over the ocean. This ensures that the average bias over the sky (Fig. 1) is removed and that the ocean Ta are unchanged on average.

\subsection{The Aquarius simulator}

The Aquarius simulator is the key tool for the calibration. It is a forward radiative transfer model that computes the antenna temperature for the three Aquarius radiometers [2]. The contributions incident on the antenna are weighted and integrated over the antenna gain patterns. The contributions from the Sky include the galaxy and cosmic background emission, the Sun and the Moon. The contributions from the Earth include emission from oceans, land and ice present in the field of view, atmospheric contributions (i.e. upward emission, attenuation of surface emission and contributions reflected at the surface such as downward atmospheric emission) and Faraday rotation that results in polarizations mixing. The simulator is used to compute $T_{a, s i m}$ in (2). This is done for the $30 \mathrm{CSC}$ and all the ocean points involved in the calibration. This is also done over reference land sites for validation purpose. The simulator is the same tool as used in the calibration over the ocean for producing V5.0.

\subsection{Celestial Sky Calibration Maneuvers}

The Celestial Sky is an important calibration target because it offers large regions of homogeneous brightness temperature (TB changes less than $0.2 \mathrm{~K}$ ), is stable in time and relatively well known. During a CSC maneuver, the spacecraft is pitched by $180^{\circ}$ for a 5 minutes period in order for the radiometers to point to the Sky and have most of the antenna power ( $\sim 97 \%)$ off the Earth surface [3]. Because there are still a few percent of power coming from the Earth, the CSC maneuvers are performed over oceans in order to minimize the sources of errors (radio frequency interferences and surface emission uncertainty). Between March 2012 and June 2015, 30 CSC maneuvers have been conducted with the beams pointing toward calm region of the Sky and the spacecraft flying over the open ocean.

\subsection{Land Validation Sites}

The comparisons over land are performed at sites monitored by the USDA. We use the Little River and Little Washita watersheds that are collocated with Aquarius middle and outer beam ground tracks respectively. Because the in situ soil moisture shows significant differences with the value derived from NCEP model that is used in the nominal Aquarius land model, it is important to perform land validations where there are in situ observations. 

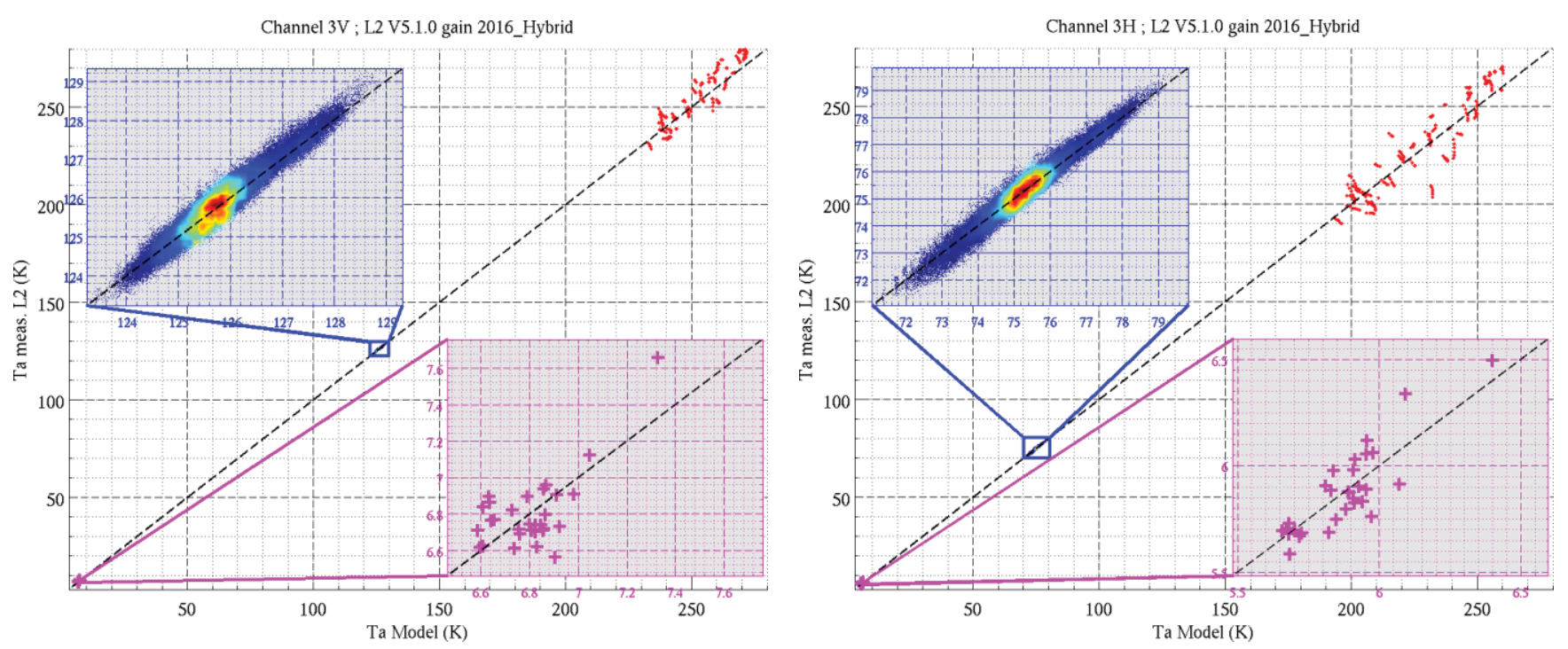

Fig. 3. $T_{a}$ measured by Aquarius versus simulated over (magenta inset) the Celestial Sky, (blue inset) ocean and (red dots) land, for the outer beam at (left) vertical and (right) horizontal polarizations. Insets are close-ups of ocean and Sky data. The Aquarius data are from V5.1, the final release with full range calibration.

\section{RESULTS OF THE FULL RANGE CALIBRATION}

The results of the linear adjustment to the calibration are reported in Table 1. The changes in slope ( $a$ parameter) are relatively small at less than 2 percent. The largest corrections, both in slope and offset ( $b$ parameter), are for channel $3 \mathrm{~V}$ which had the largest bias over the sky. For the same reason, the smallest changes are for channel $3 \mathrm{H}$ which has a very small bias over the cold sky.

Table 1: Values for the full range calibration coefficients $a$ and $b$.

\begin{tabular}{|c|c|c|c|c|}
\hline & \multicolumn{2}{|c|}{ V-pol } & \multicolumn{2}{c|}{ H-pol } \\
\hline & $a$ & $b$ & $a$ & $b$ \\
\hline B1 & 1.00335 & -0.35929 & 1.00741 & -0.65957 \\
\hline B2 & 1.00834 & -0.96556 & 1.00350 & -0.29487 \\
\hline B3 & 1.01770 & -2.23391 & 1.00131 & -0.10757 \\
\hline
\end{tabular}

\subsection{Cold Sky}

The calibration adjustment in V5.1 removes the bias over the cold Sky for all channels (Fig 1., blue curve). There are small variations in cold sky Ta between the different maneuvers, of about $0.8 \mathrm{~K}$ and they suggest that the slope at the low end of the $\mathrm{Ta}$ is in good agreement between observation and simulations (Fig. 3, magenta inset). But the dynamic range is very small so it is not possible to have an accurate validation from these data alone.

\subsection{Ocean}

The impact of the calibration on the ocean is shown in Fig. 3 for the Ta and in Fig. 4 for the salinity. Because the change in slope in the calibration is small and the dynamic range of Ta over ocean is only a few Kelvin, the impact is generally negligible. This is demonstrated by the very small changes in the average and standard deviation of SSS error in Fig. 4. It is possible that for Ta unusually far from the mean ocean Ta the impact of the calibration will be noticeable. It should be noted that the SSS retrieval algorithm uses an empirical model for correcting the impact of roughness, which has not been re-tuned for V5.1. Inconsistencies could result from the model tuned to a different calibration, and re-deriving the roughness model parameters would mitigate them.

\subsection{Land}

The impact of the recalibration on land is relatively small. At a reference land $\mathrm{Ta}$ of $250 \mathrm{~K}$, it adds $0.5 \mathrm{~K}(1 \mathrm{~V}), 1.1 \mathrm{~K}$ $(2 \mathrm{~V}), 2.2 \mathrm{~K}(3 \mathrm{~V})$ in $\mathrm{V}$-pol to the three beams respectively. In H-pol, it adds $1.2 \mathrm{~K}(1 \mathrm{H}), 0.6 \mathrm{~K}(2 \mathrm{H}), 0.2 \mathrm{~K}(3 \mathrm{H})$. These changes are small compared to the uncertainty on the model illustrated by the scatter in Fig. 2 and Fig. 3 (red dots). It is not possible to state confidently whether V5.1 is an improvement on V5.0 without additional reference data at the warm end. But it demonstrates that the bias over the cold Sky can be corrected without degrading Ta at the warm end. Further validation at the warm end is underway with the inclusion of additional instrumented land sites. Another validation target being considered is the Amazon forest. One aspect being investigated in particular is the increase in polarization ratio due to the larger correction of $3 \mathrm{~V}$ compared to $3 \mathrm{H}$.

\section{CONCLUSIONS AND DISCUSSION}

As part of the nominal processing, Aquarius antenna temperatures are calibrated empirically to radiative transfer simulations over oceans, removing a global average bias with respect to the reference ancillary field for SSS. This leaves observable biases at the low end of antenna 

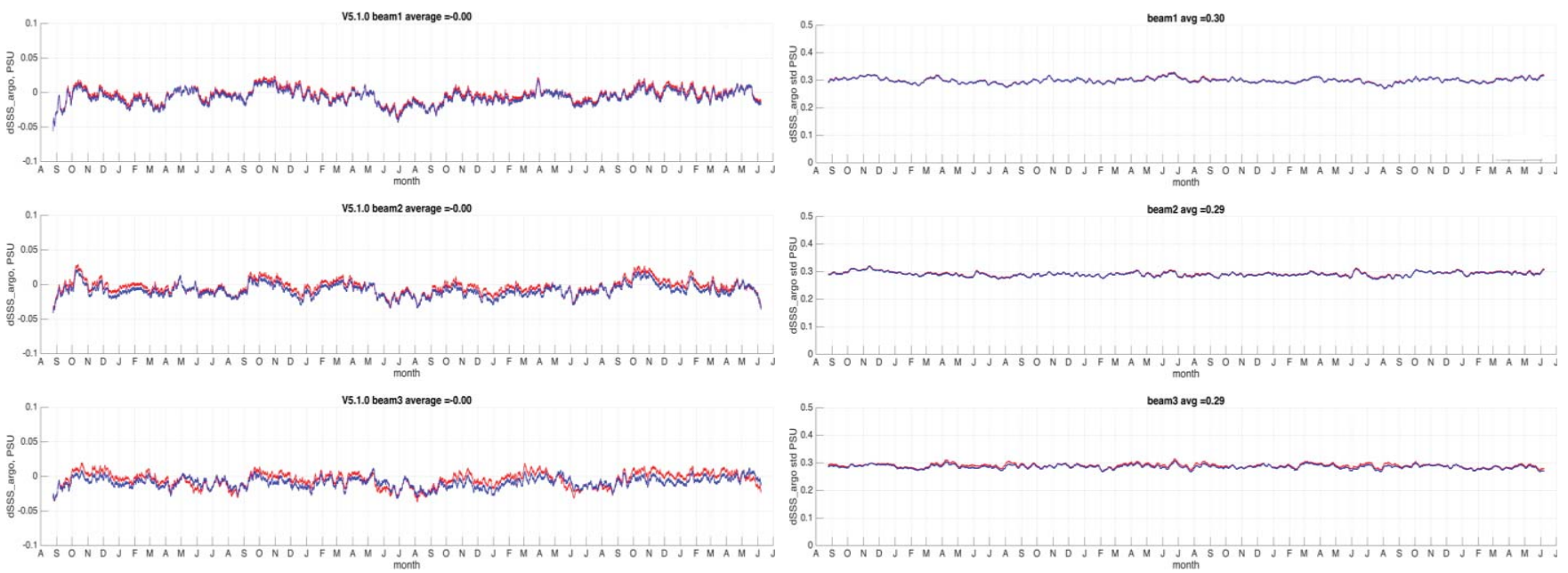

Fig. 4. Time series of (left) mean and (right) standard deviation of the difference in global SSS between Aquarius retrievals and Argo in situ observations, for Aquarius three beams (top, middle and bottom panels). The products are (blue) V5.0 and (red) V5.1. These metrics for SSS retrievals are practically unchanged by the full range calibration (V5.1). The time series covers the whole Aquarius mission duration.

temperatures when looking at the cold sky. We describe a research product with the aim of producing a calibration that covers the full range of antenna temperatures. The residual bias at the low end is corrected using a linear regression between the measured and simulated Ta over the Sky and the ocean. The performances are evaluated over the ocean and well instrumented land sites. Further validations over land are underway. We are also assessing the possible impact of residual instrument non linearity.

\section{REFERENCES}

[1] D. M. Le Vine, G. S. E. Lagerloef, and S. E. Torrusio, "Aquarius and remote sensing of sea surface salinity from space," Proc. IEEE, vol. 98, no. 5, pp. 688-703, 2010.

[2] D. M. Le Vine, E. P. Dinnat, S. Abraham, P. De Matthaeis, and F. J. Wentz, "The Aquarius simulator and cold-sky calibration," IEEE Trans. Geosci. Remote Sens., vol. 49, no. 9, pp. 3198-3210, 2011.

[3] E. P. Dinnat, D. M. Le Vine, J. R. Piepmeier, S. T. Brown, and L. Hong, "Aquarius L-band Radiometers Calibration Using Cold Sky Observations," IEEE J. Sel. Top. Appl. Earth Obs. Remote Sens., vol. 8, no. 12, pp. 5433-5449, 2015.

[4] R. Bindlish, T. Jackson, M. Cosh, T. Zhao, and P. O'Neill, "Global soil moisture from the aquarius/SAC-D satellite: Description and initial assessment," IEEE Geosci. Remote Sens. Lett., vol. 12, no. 5, pp. 923-927, 2015.

[5] L. Brucker, E. P. Dinnat, G. Picard, and N. Champollion, "Effect of snow surface metamorphism on aquarius L-band radiometer observations at Dome C, antarctica," IEEE Trans. Geosci. Remote Sens., vol. 52, no. 11, pp. 7408-7417, Nov. 2014.

[6] X. Tian-Kunze, L. Kaleschke, N. Maaß, M. Mäkynen, N. Serra, M. Drusch, and T. Krumpen, "SMOS-derived thin sea ice thickness: algorithm baseline, product specifications and initial verification," Cryosph., vol. 8, no. 3, pp. 997-1018, 2014.

[7] L. Brucker, E. P. Dinnat, and L. Koenig, "Weeklygridded Aquarius L-band radiometer/scatterometer observations and salinity retrievals over the polar regions -- Part 2: Initial product analysis," Cryosph., vol. 8, no. 3, pp. 915-930, 2014. 